\title{
Eficácia das injeções de toxina botulínica no tratamento da hiperidrose primária: uma meta análise
}

\author{
Effectiveness of botulinic toxin injections in the treatment of primary hyperhidrosis: a meta- \\ analysis \\ Efectividad de las inyecciones de toxina botulínica en el tratamiento de la hiperhidrosis \\ primaria: un metanálisis
}

Sonia Oliveira Lima ${ }^{1 *}$, Rafael Silva Santos ${ }^{1}$, Ana Karina Rocha Hora Mendonça ${ }^{1}$, Arthur Valido Deda $^{1}$, Vivian Fernandes dos Santos ${ }^{1}$, Renata Lima Batalha de Andrade ${ }^{1}$, Maria Bernadete Galrão de Almeida Figueiredo ${ }^{1}$, Fabiana Pereira Guimarães Brito ${ }^{1}$, Marco Antonio Prado Nunes ${ }^{2}$, Carla Viviane Freitas de Jesus'.

\section{RESUMO}

Objetivo: Avaliar a eficácia da aplicação de toxina botulínica no tratamento da hiperidrose primária em seus diferentes locais de acometimento. Métodos: Realizou-se uma meta-análise através dos bancos de dados PubMed, Cochrane, Scopus, Scientific Electronic Library Online (SciELO) e Open Thesis. Os descritores utilizados foram "hiperidrose primária", "tratamento" e "toxina botulínica". Dois revisores realizaram protocolos independentes usando um formulário padrão, a partir de um protocolo de revisão sistemática pré-definido, PRISMA. Resultados: O protocolo de busca encontrou 1096 artigos na seleção inicial, destes foram incluídos 14 estudos. A análise de homogeneidade mostrou uma Cochran Teste $Q$ de 80,1242 ( $p=0,001)$ e teste de Higgins $I^{2}$ de $96,24 \%$, A simetria foi avaliada pelo teste de assimetria do gráfico de funil com um valor de $t$ de 2,9111 ( $p=0,0131$ ). Os dados sobre eventos adversos foram assimétricos e heterogêneos, justificando o uso do modelo de efeitos fixos. Considerações finais: A utilização de toxina botulínica é segura e efetiva para o tratamento da Hiperidrose Primária (Nível de Evidência 1b). Injeções de toxinas atuam como uma medida paliativa, promovendo a cessação ou redução do suor com duração de efeito extremamente variável de 4 semanas a 6 meses, e com poucos efeitos adversos.

Palavras-chave: Hiperidrose, Toxinas botulínicas, Tratamento.

\begin{abstract}
Objective: To evaluate the effectiveness of applying botulinum toxin in the treatment of primary hyperhidrosis in its different sites of involvement. Methods: A meta-analysis was performed using the PubMed, Cochrane, Scopus, Scientific Electronic Library Online (SciELO) and Open Thesis databases. The keywords used were "primary hyperhidrosis", "treatment" and "botulinum toxin". Two reviewers performed independent protocols using a standard form, based on a pre-defined systematic review protocol, PRISMA. Results: The search protocol found 1096 articles in the initial selection, of which 14 studies were included. Homogeneity analysis showed a Cochran Test $Q$ of $80.1242(p=0.001)$ and a Higgins $I^{2}$ test of $96.24 \%$. Symmetry was assessed by the asymmetry test of the funnel graph with a t-value of 2.9111 ( $p=0.0131)$. The data on adverse events were asymmetric and heterogeneous, justifying the use of the fixed effects model. Final Considerations: The use of botulinum toxin is safe and effective for the treatment of Primary Hyperhidrosis (Evidence Level 1b).
\end{abstract}

${ }^{1}$ Universidade Tiradentes (UNIT), Aracaju - SE. *E-mail: sonialima.cirurgia@gmail.com

2 Universidade Federal de Sergipe (UFS), Aracaju - SE.

SUBMETIDO EM: $8 / 2020$

ACEITO EM: 9/2020

PUBLICADO EM: 12/2020

REAS/EJCH | Vol. 12(12) | e5092 | DOI: https://doi.org/10.25248/reas.e5092.2020 Página 1 de 11 
Injections of toxins act as a palliative measure, promoting the cessation or reduction of sweat with an extremely variable duration of effect from 4 weeks to 6 months, and with few adverse effects.

Keywords: Hyperhidrosis, Botulinum toxins, Treatment.

\section{RESUMEN}

Objetivo: Evaluar la efectividad de la aplicación de toxina botulínica en el tratamiento de la hiperhidrosis primaria en sus diferentes sitios de afectación. Métodos: Se realizó un metaanálisis utilizando las bases de datos PubMed, Cochrane, Scopus, Scientific Electronic Library Online (SciELO) y Open Thesis. Las palabras clave utilizadas fueron "hiperhidrosis primaria", "tratamiento" y "toxina botulínica". Dos revisores realizaron protocolos independientes utilizando un formulario estándar, basado en un protocolo de revisión sistemática predefinido, PRISMA. Resultados: El protocolo de búsqueda encontró 1096 artículos en la selección inicial, de los cuales se incluyeron 14 estudios. El análisis de homogeneidad mostró una prueba $Q$ de Cochran de 80,1242 ( $p=0,001$ ) y una prueba de Higgins $\mathrm{I}^{2}$ de $96,24 \%$. La simetría se evaluó mediante la prueba de asimetría del gráfico de embudo con un valor t de 2,9111 $(p=0,0131)$. Los datos sobre eventos adversos fueron asimétricos y heterogéneos, lo que justifica el uso del modelo de efectos fijos. Consideraciones finales: El uso de toxina botulínica es seguro y eficaz para el tratamiento de la hiperhidrosis primaria (nivel de evidencia 1b). Las inyecciones de toxinas actúan como medida paliativa, favoreciendo el cese o la reducción del sudor con una duración de efecto extremadamente variable de 4 semanas a 6 meses, y con pocos efectos adversos.

Palabras clave: Hiperhidrosis, Toxinas botulínicas, Tratamiento.

\section{INTRODUÇÃO}

A derme possui terminações nervosas, vasos sanguíneos, glândulas sudoríparas écrinas e apócrinas, que são distribuídas pelo corpo humano. As glândulas écrinas encontram-se em maior número e são mais desenvolvidas nas regiões facial, palmar, plantar e axilar, liberam o suor, fluído incolor e inodoro, composto principalmente por água $(99,5 \%)$ e por sais como sódio, potássio, cálcio, magnésio, cloro, uréia e lactato. As glândulas apócrinas são responsáveis pelo odor e têm sua função iniciada na puberdade, liberando secreções compostas por substâncias lipídicas que são excretadas sobre estímulo emocional. O suor é indispensável na termorregulação do corpo, e seu excesso é conhecido como hiperidrose, que pode ser primária ou secundária.

A hiperidrose secundária, é generalizada pelo corpo, pode ocorrer em repouso ou no sono, sendo causada por situações como atividade física, aumento da temperatura corporal, menopausa, condições como hipertireoidismo, obesidade, efeito colateral de medicações, tipos de alimento entre outras (WEBER A, et al., 2005; SOLISH N, et al., 2007; SCHNEIER FR, et al., 2012).

A Hiperidrose Primária (HP) é localizada, dificilmente ocorre no repouso ou no sono, e excede as necessidades da termoregulação do corpo, podendo ser associada a bromoidrose, causar prejuízo na qualidade de vida por inteferir nas atividades diárias, laborais e sociais (DIAS LIDEN, et al., 2016). É sabido que não há diferença entre o número e a distribuição das glândulas écrinas pelo corpo, entre pacientes com ou sem hiperidrose primária, porém essas glândulas, apresentam maior atividade em indivíduos com HP, por provável desregulação entre o sistema nervoso simpático e parassimpático.

Estudos genéticos mostraram que o cromossomo $14 q$ está possivelmente associado com a hiperidrose primária, e devido seu carácter autossômico dominante, o histórico familiar é positivo em grande parte dos acometidos (WEBER A, et al., 2005; LAKRAJ AAD, et al., 2013).

A HP acomete homens e mulheres, atingindo cerca de $3 \%$ da população mundial, e em grupos específicos ela varia de $0,6 \%$ em Israel a $18 \%$ no Brasil (ADAR R, 1987; LIMA SO, et al., 2019). O início dos sintomas, é mais prevalente na infância e adolescência, surgindo em situações de estresse, podendo comprometer a autoestima, atividades diárias, laborais e sociais (SCHNEIER FR, et al., 2012). 
Fenili R, et al. (2009) afirmam que o diagnóstico de HP é essencialmente clínico, podendo ser confirmado com os critérios diagnósticos e a escala mais aceita para avaliar a gravidade da doença é a Hyperhidrosis Disease Severity Scale (HDSS). Essa escala vai desde a sudorese imperceptível, que não afeta as atividades diárias (HDSS-1), à sudorese intolerável sempre interferindo nas atividades diárias (HDSS-4) (SOLISH N, et al., 2007).

Entre os exames complementares utilizados para diagnóstico e quantificação da HP são o Minor Test e gravimetria (MINOR V, et al., 1928; HOORENS I e ONGENAE K, 2012). Para reduzir a transpiração na região afetada, têm sido utilizado tratamento clínico ou cirúrgico. Dentre estes, incluem drogas ansiolíticas, simpaticolíticas, cloreto de alumínio, iontoforese, lipoaspiração, extipações de glândulas sudoríparas, curetagem do tecido subcutâneo da região axilar e simpatectomias toracoscópica e/ou retroperiteneotoscópica, ablação química ou por radiofrequência e aplicação de toxinas.

Dentre as opções terapeuticas à aplicação da toxina botunílica (TB) possui caráter não cirúrgico, podendo ser realizada em nível ambulatorial sendo segura e factível sua reaplicação (BARONCELLO JB, et al., 2014; ASIK ZS, et al., 2008; VASCONCELLOS RC, et al., 2019).

A toxina botulínica é uma neurotoxina, derivada da bactéria Clostridium botulinum bacilo gram-positivo, anaeróbico, produtora de esporos (LAKRAJ AAD, et al., 2013). Há mais de uma década vem sendo utilizada nos tratamentos estéticos, com baixos efeitos colaterais como cefaléia, fraqueza muscular, dor no local da aplicação e diminuição da saliva. Pode ser, também, indicada no tratamento de diversas doenças nas especialidades oftalmológica, neurológica, urológica e dermatológica.

Em 2002, começou a ser empregada no controle da hiperidrose primária, sendo esse tratamento aprovado pelo Food and Drug Administration (FDA), em 2004. Seu mecanismo de ação consiste no bloqueio temporario, da liberação de neurotransmissores responsáveis pela contração muscular inibindo, principalmente, a exocitose de acetilcolina na fenda pré-sináptica das glândulas écrinas envolvidas na produção do suor. A TB é contra-indicada em pacientes alérgicos, gestantes, lactantes, crianças menores de 2 anos, idosos acima de 65 anos e portadores de doenças neuromusculares (GOLAN DE, et al., 2009).

$O$ uso da TB é um método seguro, preciso e bem tolerado, porém sua ação permanece por aproximadamente três a seis meses, podendo ser replicado (SCHLESSINGER J, et al., 2017). Portanto este estudo objetivou realizar uma meta-análise para avaliar a eficácia da toxina botulínica no tratamento da hiperidrose primária em seus diferentes locais de acometimento.

\section{MÉTODOS}

O protocolo de triagem realizou pesquisas independentes, de abril de 2018 a julho de 2020. Os bancos de dados de pesquisa incluíram PubMed, Cochrane, Scopus, Scientific Electronic Library Online (SciELO) e Open Thesis. Os descritores utilizados para construir a estratégia de busca foram "hiperidrose primária", "tratamento" e "toxina botulínica". Dois revisores realizaram protocolos independentes usando um formulário padrão, a partir de um protocolo de revisão sistemática pré-definido, PRISMA (PATI D e LORUSSO LN, 2018).

O PRISMA foi atualizado em 2015, com o objetivo de facilitar o desenvolvimento e a geração de relatórios de protocolos de revisão sistemática, facilitando replicabilidade dos estudos e aprimorando a validade aparente, facilitando a criação de protocolos de revisões prospectivos. Os pesquisadores fizeram uma tentativa de contato via e-mail para recuperar artigos de acesso restrito. Os autores que não responderam no prazo de 15 dias foram excluídos da meta-análise. Foram recuperadas as referências bibliográficas de cada artigo para encontrar possíveis estudos elegíveis.

Para ser elegível, os estudos devem ter desenho prospectivo e métodos aleatórios; os pacientes com hiperidrose primária devem ter seu regime de tratamento usando, exclusivamente, tratamento com toxina botulínica ou uma comparação de toxina botulínica versus grupos placebo ou grupos cirúrgicos. Um terceiro revisor estava disponível para resolver possíveis divergências. Estudos de revisão, relatos de casos, séries de casos e estudos observacionais, quando encontrados, foram excluídos. Os principais resultados medidos nesta revisão incluem tipo de estudo, nível de evidência, localização da hiperidrose, formulação e dosagens de medicamentos, tempo de seguimento, taxa de sucesso, efeitos adversos, taxa de abandono. 
Para garantir a qualidade dos estudos, utilizou-se a escala de Jadad para randomized controlled trials (RCTs), avaliação de randomização, mascaramento e formulário de consentimento informado e taxa de abandono e estudos de alta qualidade obtidos $\geq 3$ pontos. A análise de homogeneidade mostrou um teste $Q$ de Cochran de $80.1242(p=0.001)$ e um teste $l^{2}$ de Higgins de $96.24 \%$. A simetria foi avaliada pelo teste de assimetria do gráfico de funil com valor t de $2.9111(p=0.0131)$.

Portanto, os dados sobre eventos adversos foram assimétricos e heterogêneos (Figura 1). O nível de significância adotado foi de 0,05. A metanálise utilizou o STATA 15.0, R Core Team 3.4.1 e o pacote "metafor" (VIECHTBAUER W, 2010).

\section{RESULTADOS}

O protocolo de busca encontrou 568 estudos no PubMed, 4 no SciELO, 452 no Scopus, 72 no Cochrane, totalizando 1096 artigos na seleção inicial, publicados de julho de 1993 a julho de 2020. Após a exclusão de duplicatas, 614 referências tiveram seus títulos e resumos analisados, com 490 exclusões. Após a verificação dos critérios de inclusão para os estudos, 62 tiveram uma triagem em texto completo e 25 foram considerados como estudos potencialmente relevantes para serem incluídos na revisão sistemática. A busca manual com referência cruzada não identificou mais estudos, e após a extração dos dados, 14 foram definidos para a metanálise final (Figura 1).

Figura 1 - Fluxograma PRISMA para seleção de artigos publicados no período de julho de 1993 a julho de 2020.

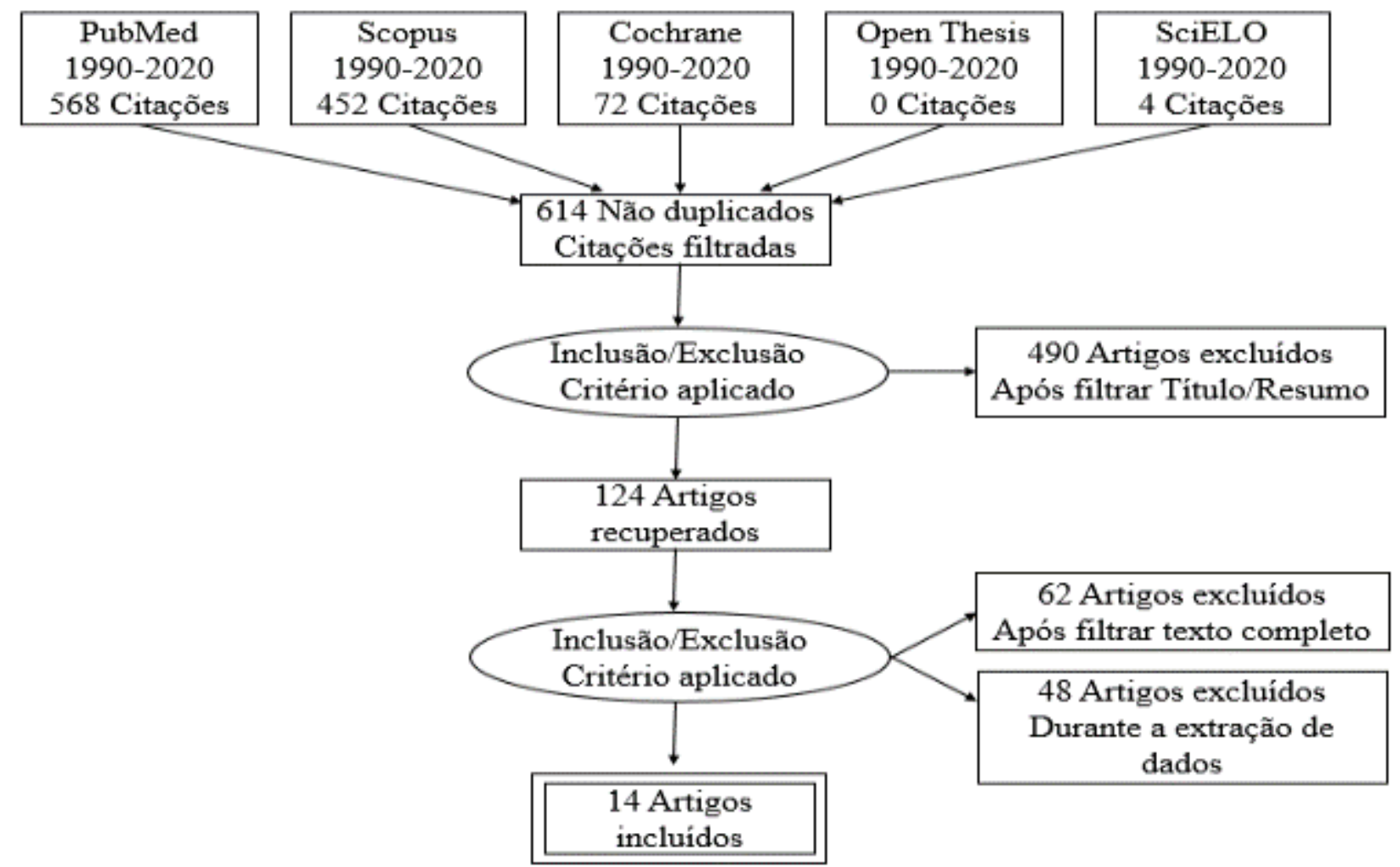

Fonte: Lima SO, et al., 2020.

O número de participantes, em cada estudo, variou de 8 a 322, com intervalo de idade que variou de 16 a 80 anos. Os estudos incluíram pacientes com hiperidrose primária nos sítios axilar, palmar, frontal e plantar, de ambos os sexos. A análise de homogeneidade mostrou um teste Cochran $Q$ de $80.1242(p=0,001)$ e um teste Higgins $\mathrm{I}^{2}$ de $96,24 \%$. A simetria foi avaliada pelo teste de assimetria do gráfico de funil com um valor de $t$ de 2,9111 ( $p=0,0131$ ) (Figura 2). Portanto, os dados sobre eventos adversos foram assimétricos e heterogêneos, justificando o uso do modelo de efeitos fixos (Figura 3). 
Figura 2 - Gráfico de funil de estudos em indivíduos que experimentaram o evento adverso.

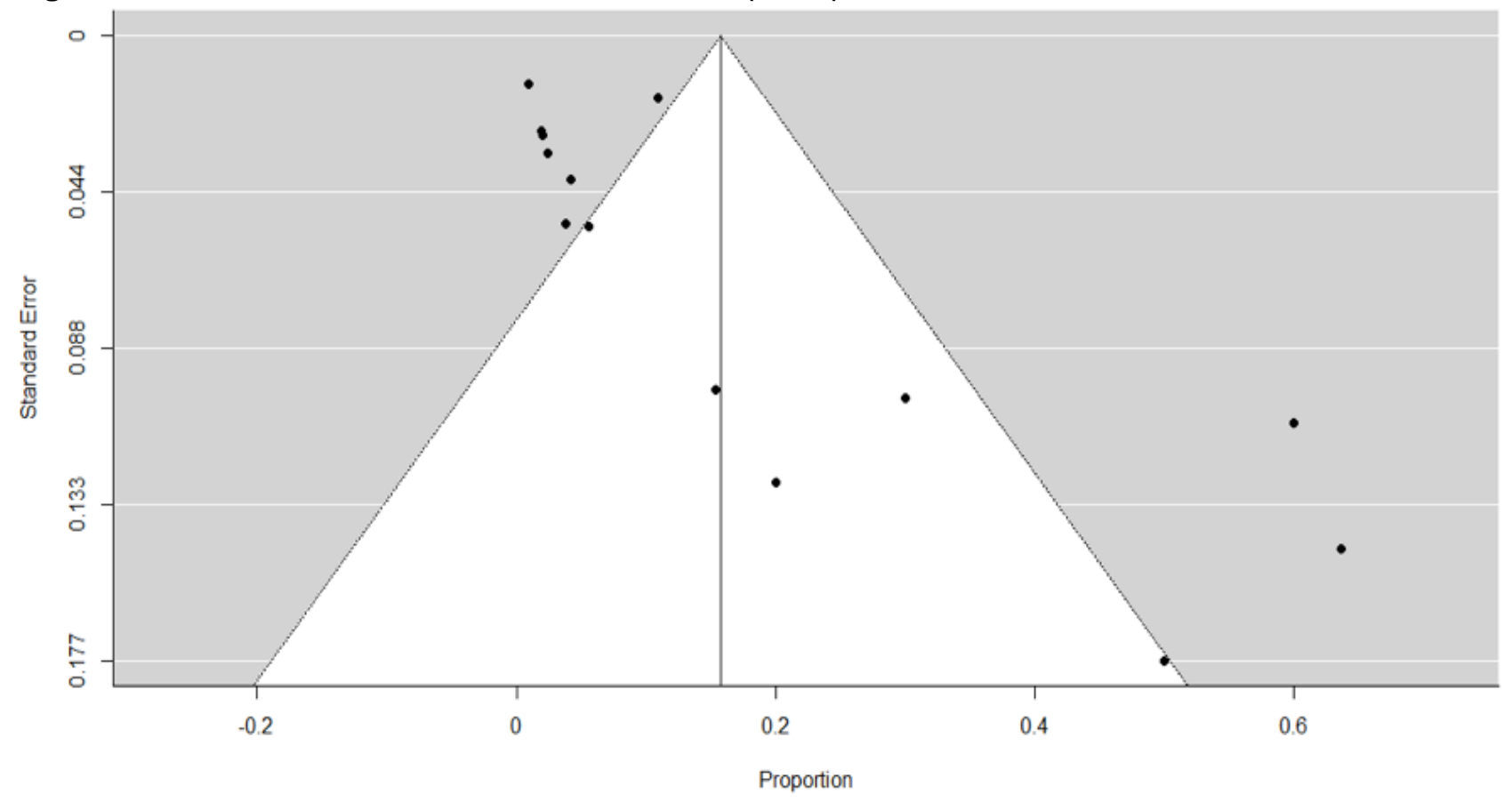

Fonte: Lima SO, et al., 2020.

Figura 3 - Gráfico de floresta com a proporção de indivíduos que sofrem o evento adverso, seus intervalos de confiança e a medida metanalítica.

Autor

Proporção [95\% cl]

\begin{tabular}{lll}
\hline Jee & Campanati & \\
Dressler \\
Ibrahim \\
Glogau
\end{tabular}

Fonte: Lima SO, et al., 2020.

O resultado meta-analítico mostra uma proporção de eventos adversos de $5 \%$, com um intervalo que pode ir de $3 \%$ a $7 \%$ e significância $(p<0,05)$ (figura 3$)$. Os resultados de cada estudo foram resumidos e os autores foram ordenados por ano de publicação. Foram avaliados 576 pacientes, com indicação de tratamento com toxina botulínica, com resolução temporária dos sintomas para todas as dosagens. Todas as dosagens levaram os pacientes a terem uma redução significativa do suor (Quadro1). 
Quadro 1 - Resumo dos resultados. Pesquisa atualizada em 4 de julho, triagem das bases de dados: PubMed, Scopus, SciELO, Cochrane.

\begin{tabular}{|c|c|c|c|c|c|}
\hline Autor/Ano & $\begin{array}{l}\text { Número de } \\
\text { pacientes }\end{array}$ & Desenho do estudo & Droga e dosagem & Resultado primário & Efeitos colaterais \\
\hline \multirow{2}{*}{$\begin{array}{l}\text { Jee SA, et al. } \\
\qquad(2015)\end{array}$} & \multirow[t]{2}{*}{24} & \multirow{2}{*}{$\begin{array}{l}\text { Ensaio Clínico } \\
\text { Randomizado }\end{array}$} & Onabotulinumtoxin A 50MU & Redução do Suor / Cura & \multirow[t]{2}{*}{ Dor transitória } \\
\hline & & & Rimabotulinumtoxin B 1500MU & Redução do Suor / Cura & \\
\hline \multirow{2}{*}{$\begin{array}{l}\text { Campanati } A \text {, et } \\
\text { al. (2014) }\end{array}$} & \multirow{2}{*}{25} & \multirow{2}{*}{$\begin{array}{l}\text { Ensaio Clínico } \\
\text { Randomizado }\end{array}$} & Incobotulinumtoxin A 100-150MU & Redução do Suor / Cura & \multirow{2}{*}{ Sem relatos } \\
\hline & & & Onabotulinumtoxin A 100-150MU & Redução do Suor / Cura & \\
\hline \multirow{2}{*}{$\begin{array}{l}\text { Dressler D e Adib } \\
\text { Saberi F (2013) }\end{array}$} & \multirow{2}{*}{51} & \multirow{2}{*}{$\begin{array}{l}\text { Ensaio Clínico } \\
\text { Randomizado }\end{array}$} & Onabotulinumtoxin A 50MU & Redução do Suor / Cura & \multirow{2}{*}{ Sem relatos } \\
\hline & & & Onabotulinumtoxin A 100MU & Redução do Suor / Cura & \\
\hline \multirow{2}{*}{$\begin{array}{l}\text { Ibrahim O, et al. } \\
\qquad(2013)\end{array}$} & \multirow{2}{*}{20} & \multirow{2}{*}{$\begin{array}{l}\text { Ensaio Clínico } \\
\text { Randomizado }\end{array}$} & Onabotulinumtoxin-A 100MU & Redução do Suor / Cura & Sem relatos \\
\hline & & & Suction-Curettage & Redução do Suor / Cura & $\begin{array}{l}\text { Dor transitória, neuralgia transitória, } \\
\text { hiperpigmentação }\end{array}$ \\
\hline \multirow{2}{*}{$\begin{array}{l}\text { Rystedt A, et al. } \\
\qquad(2013)\end{array}$} & \multirow{2}{*}{20} & \multirow{2}{*}{$\begin{array}{l}\text { Ensaio Clínico } \\
\text { Randomizado }\end{array}$} & Onabotulinumtoxin-A 100MU & Redução do Suor / Cura & \multirow{2}{*}{$\begin{array}{l}\text { Sensação de ardor local e secura } \\
\text { excessiva da pele }\end{array}$} \\
\hline & & & Abobotulinumtoxin 100 to $200 \mathrm{MU}$ & Redução do Suor / Cura & \\
\hline \multirow{2}{*}{$\begin{array}{l}\text { Glogau RG } \\
\text { (2007) }\end{array}$} & \multirow[t]{2}{*}{12} & \multirow{2}{*}{$\begin{array}{l}\text { Ensaio Clínico } \\
\text { Randomizado }\end{array}$} & Onabotulinumtoxin-A 100MU & Redução do Suor / Cura & Sem relatos \\
\hline & & & Placebo & Sem efeito & Foliculite, eritema, eczema \\
\hline
\end{tabular}

REAS/EJCH | Vol. 12(12) | e5092 | DOI: https://doi.org/10.25248/reas.e5092.2020 Página 6 de 11 
Revista Eletrônica Acervo Saúde / Electronic Journal Collection Health | ISSN 2178-2091

\begin{tabular}{|c|c|c|c|c|c|}
\hline Autor/Ano & $\begin{array}{l}\text { Número de } \\
\text { pacientes }\end{array}$ & Desenho do estudo & Droga e dosagem & Resultado primário & Efeitos colaterais \\
\hline \multirow{2}{*}{$\begin{array}{l}\text { Talarico-Filho S, } \\
\text { et al. (2007) }\end{array}$} & \multirow{2}{*}{10} & \multirow{2}{*}{$\begin{array}{l}\text { Ensaio Clínico } \\
\text { Randomizado }\end{array}$} & Onabotulinumtoxin-A 50MU & Redução do Suor / Cura & \multirow{2}{*}{ Sudorese compensatória } \\
\hline & & & Abobotulinumtoxin 150MU & Redução do Suor / Cura & \\
\hline \multirow{2}{*}{$\begin{array}{l}\text { Almeida ART, et } \\
\text { al. (2007) }\end{array}$} & \multirow{2}{*}{20} & \multirow{2}{*}{$\begin{array}{l}\text { Ensaio Clínico } \\
\text { Randomizado }\end{array}$} & Onabotulinumtoxin-A 50MU & Redução do Suor / Cura & \multirow{2}{*}{$\begin{array}{l}\text { Inibição leve da contração muscular } \\
\text { da testa }\end{array}$} \\
\hline & & & Abobotulinumtoxin 150MU & Redução do Suor / Cura & \\
\hline \multirow{2}{*}{$\begin{array}{l}\text { Moreau MS, et al. } \\
\qquad(2003)\end{array}$} & \multirow[b]{2}{*}{8} & \multirow{2}{*}{$\begin{array}{l}\text { Ensaio Clínico } \\
\text { Randomizado }\end{array}$} & Onabotulinumtoxin-A 69MU & Redução do Suor / Cura & $\begin{array}{l}\text { Fraqueza de pinça de índice de } \\
\text { polegar transitório }\end{array}$ \\
\hline & & & Abobotulinumtoxin 283MU & Redução do Suor / Cura & $\begin{array}{l}\text { Fraqueza transitória da pinça do } \\
\text { índice do polegar, Peso do membro } \\
\text { superior direito }\end{array}$ \\
\hline \multirow{2}{*}{$\begin{array}{l}\text { Odderson IR } \\
\quad(2002)\end{array}$} & \multirow{2}{*}{18} & \multirow{2}{*}{$\begin{array}{l}\text { Ensaio Clínico } \\
\text { Randomizado }\end{array}$} & Onabotulinumtoxin-A 100MU & Redução do Suor / Cura & Sudorese compensatória leve \\
\hline & & & Placebo & Sem efeito & - \\
\hline \multirow{2}{*}{$\begin{array}{l}\text { Saadia D, et al. } \\
\qquad(2001)\end{array}$} & \multirow{2}{*}{24} & \multirow{2}{*}{$\begin{array}{l}\text { Ensaio Clínico } \\
\text { Randomizado }\end{array}$} & Onabotulinumtoxin-A 50MU & Redução do Suor / Cura & \multirow{2}{*}{$\begin{array}{l}\text { Fraqueza de pinça de índice de } \\
\text { polegar transitório }\end{array}$} \\
\hline & & & Onabotulinumtoxin-A 100MU & Redução do Suor / Cura & \\
\hline \multirow{2}{*}{$\begin{array}{l}\text { Naumann M e } \\
\text { Lowe NJ (2001) }\end{array}$} & \multirow{2}{*}{320} & \multirow{2}{*}{$\begin{array}{l}\text { Ensaio Clínico } \\
\text { Randomizado } \\
\text { Multicêntrico }\end{array}$} & Onabotulinumtoxin-A 50MU & Redução do Suor / Cura & Suor compensatório, dor transitória \\
\hline & & & Placebo & Sem efeito & Dor transitória \\
\hline \multirow{2}{*}{$\begin{array}{l}\text { Schnider P, et al. } \\
(1999)\end{array}$} & \multirow[t]{2}{*}{13} & \multirow{2}{*}{$\begin{array}{l}\text { Ensaio Clínico } \\
\text { Randomizado }\end{array}$} & Onabotulinumtoxin-A 200MU & Redução do Suor / Cura & $\begin{array}{l}\text { Prurido leve transitório, constipação } \\
\text { leve, sudorese compensatória }\end{array}$ \\
\hline & & & Placebo & Sem efeito & - \\
\hline \multirow{2}{*}{$\begin{array}{l}\text { Schnider P, et al. } \\
\text { (1997) }\end{array}$} & \multirow[t]{2}{*}{11} & \multirow{2}{*}{$\begin{array}{l}\text { Ensaio Clínico } \\
\text { Randomizado }\end{array}$} & Onabotulinumtoxin-A 200MU & Redução do Suor / Cura & $\begin{array}{l}\text { Fraqueza muscular transitória local e } \\
\text { parestesia transitória, dor transitória }\end{array}$ \\
\hline & & & Placebo & Sem efeito & Hematomas menores \\
\hline Total & 576 & & & & \\
\hline
\end{tabular}

Fonte: Lima SO, et al., 2020.

REAS/EJCH | Vol. 12(12) | e5092 | DOI: https://doi.org/10.25248/reas.e5092.2020 Página 7 de 11 
Verificou-se que a técnica utilizada para a injeção de toxina foi semelhante em todos os estudos, consistindo de uma injeção intradérmica de $0,1 \mathrm{ml}$ a $0,2 \mathrm{ml}$ de toxina, cada aplicação com $1,5 \mathrm{a} 2 \mathrm{~cm}$ uma da outra (Figura 4).

Figura 4 - Protocolo de espaçamento de infiltração de toxina observado nos estudos.

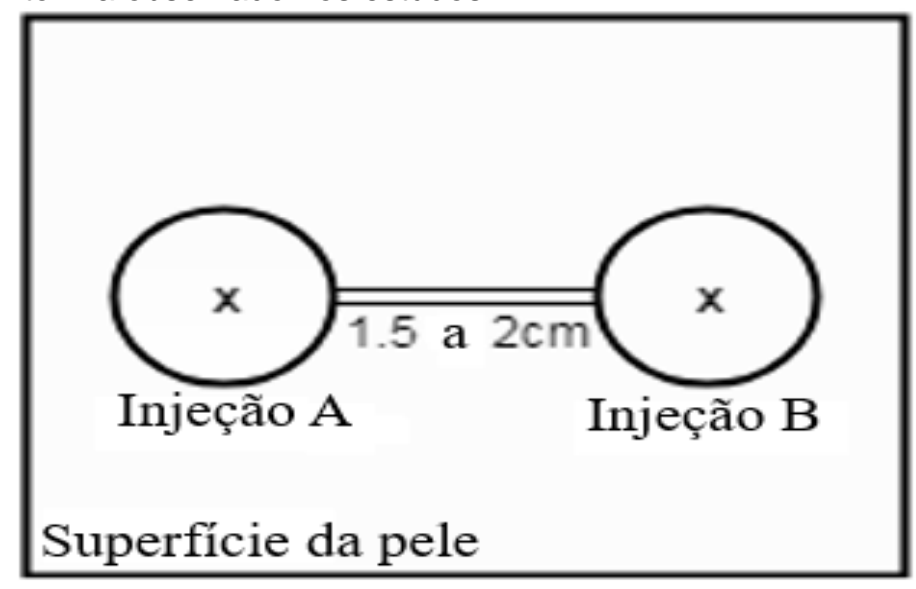

Fonte: Lima SO, et al., 2020.

\section{DISCUSSÃO}

O excesso de transpiração nem sempre é reconhecido como um sinal de doença, tanto pelos portadores, familiares e como pelos profissionais de saúde, e que pode ser tratada. A severidade do suor pode levar a problemas biopsicossociais, desconforto e incapacidades, com relatos na literatura de ansiedade e depressão, altos índices de isolamento e baixa qualidade de vida (WEBER A, et al., 2005; DIAS LIDEN, et al., 2016). A hiperidrose primária pode ocorrer em sítios isolados ou combinados, sendo a palmar, geralmente associada à plantar, e seu início ocorre na infância e adolescência. Esta sudorese pode levá-los a problemas escolares como dificuldades para escrever, segurar material escolar, e por molhar as estruturas que são manuseadas.

Seus portadores são repreendidos pelos educadores que, muitas vezes, desconhecem essa doença, e no ponto de vista social evitam apertos de mão e utilizam, constantemente, toalhas ou a própria roupa para enxugar as mãos. Estes, profissionalmente, sentem dificuldades na relação interpessoal ou em trabalhos que necessitam do manuseio, podendo causar corrosão de aparelhos metálicos, riscos de acidentes, e de danificar documentos importantes.

$\mathrm{Na}$ hiperidrose plantar, o uso de sandálias acumula suor e poeira, estragando rapidamente os calçados, e ao usar sapatos fechados com intuito de maior conforto e equilíbrio reduzindo escorregões, entorses e quedas, predispõe a marceração da pele, predispondo o surgimento de micoses e proliferação bacteriana, aumentando a bromoidrose. Nas mulheres soma-se a dificuldade de usar salto alto no exercício de determinadas profissões, como modelo e recepcionista (LIMA SO, et al., 2020; LIMA SO, et al., 2019).

A hiperidrose axilar tem início, principalmente, na puberdade com o aumento dos hormônios sexuais, e geralmente associa-se à bromoidrose e à cromoidrose, impregnando as roupas pelo suor e odor, danificandoas. Os pacientes são estigmatizados por aparentarem má higiene e, por isso, evitam o uso de roupas coloridas, preferindo roupas brancas ou pretas, e utilizam como artifício o uso de absorventes axilares. Esses transtornos, em época de puberdade, e de formação da personalidade, período de maior instabilidade emocional, aumentam a baixa estima desses adolescentes.

Na hiperidrose crâniofacial o suor da região frontal e do couro cabeludo dá um aspecto de cansaço e insegurança, dificultam o uso de maquiagem, protetor solar e outros cosméticos. Laborais se sentem incomodados pela presença do suor gotejante, a exemplo de professores, aeromoças, recepcionistas, médicos, dentistas, psicólogos e modelos (LIMA SO, et al., 2020; LIMA SO, et al., 2019).Devido à alta incidência e do melhor conhecimento da HP como doença crônica e tratável, tornou-se crescente a procura de alternativas terapêuticas clínicas ou cirúrgicas. 
Dentre as não cirúrgicas, estão o uso de drogas ansiolíticas, simpaticolíticas, cloreto de alumínio, iontoforese e aplicação de toxinas. O uso do cloreto de alumínio pode ser muito irritante e pouco eficiente. $\mathrm{O}$ tratamento oral com drogas anticolinérgicas, como a oxibutinina promove redução significativa da hiperidrose, porém com efeitos sistêmicos adversos como boca seca, cefaléia e sonolência, o que leva, por vezes, o abandono do tratamento.

A iontoforese deriva da passagem de corrente elétrica através da água na pele intacta, o seu mecanismo ainda incerto mas parece que a corrente elétrica evita a ação da glândula sudorípara, talvez por induzir uma hiperqueratose dos poros dessas glândulas interrompendo os canais iônicos secretórios dos glomerulus e obstruindo-os (LOUREIRO M, et al., 2018). Entre as técnicas definitivas incluem as diferentes modalidades de simpatectomia torácica e simpatectomia lombar, com taxas de resolução acima de 90\% (RIEGER R, 2016).

O presente estudo avaliou a eficácia da toxina botulínica no tratamento da hiperidrose primária em seus diferentes locais de acometimento. Dentre os sete tipos de toxina botulínica conhecidas (A, B, C, D, E, F, G), os dois tipos mais utilizados na terapêutica médica, são A e B. A toxina botulínica é popularmente conhecida como Botox $\circledast$ e tem efeito inibidor na liberação da acetilcolina, acredita-se que o bloqueio sináptico provocado possa promover atrofia e involução das glândulas sudoríparas.

A aplicação da toxina botulínica requer múltiplas injeções com espaço de 1,5 a 2cm entre eles (RYSTEDT A, et al., 2013; GLOGAU RG, 2007; TALARICO-FILHO S, et al., 2007; NAUMANN M e LOWE NJ, 2001; SCHNIDER P, et al., 1999; SCHNIDER P, et al., 1997). A maioria dos estudos utilizou o teste Minor, e na avaliação da intensidade do suor, o HDSS e a gravimetria. O teste de Minor é utilizado para determinar a área exata da hiperidrose, servindo de guia para os sítios onde serão aplicadas a injeção da toxina botulínica, apesar de não ser um teste quantitativo, ele é muito importante para acompanhar a eficácia do tratamento. $O$ teste consiste na aplicação de solução iodada a $2 \%$, e posteriormente, é polvilhado amido sobre a área a ser avaliada.

Assim a área hiperidrótica solubiliza o iodo, o que favorece uma reação de complexação com o amido, à medida que a reação vai acontecendo a cor castanho claro da solução iodada se transforma em azul-escuro, onde pode-se observar as áreas hipersudorética (ROMERO RF, et al., 2016; MINOR V, 1928). A aplicação da injeção de toxina botulínica, provoca dor que foi avaliada de forma objetiva, e alguns estudos utilizaram uma escala de dor visual. Com a finalidade de minimizar a dor da região axillar, pode ser utilizado o uso tópico de lidocaína, no entanto em regiões mais sensíveis como a palma da mão ou planta do pé a melhor escolha é o bloqueio neural que requer treinamento específico devido a riscos de lesão neural e graves efeitos adversos, como choque anafilático.

Outra opção é a utilização da crioteratia associada a anestesia local com lidocaína nos pontos onde serão aplicados o botox. Em todos os estudos, o uso de anestesia local ou bloqueio anestésico, antes da aplicação da toxina, causou a interrupção da dor (JEE SA, et al., 2015; CAMPANATI A, et al., 2014; DRESSLER D, et al., 2013; IBRAHIM O, et al., 2013; RYSTEDT A, et al., 2013; ALMEIDA ART, et al., 2007; GLOGAU RG, 2007; TALARICO-FILHO S, et al., 2007; MOREAU MS, et al., 2003; ODDERSON IR, 2002; SAADIA D, et al., 2001 NAUMANN M e LOWE NJ, 2001; SCHNIDER P, et al., 1999; SCHNIDER P, et al., 1997).

Diferentes doses de toxina botulínica foram observadas em estudos comparando Onabotulinumtoxin-A (ONA) versus Abobotulinuntoxin $A(A B O)$, e estudos comparando as toxinas versus placebo. Não foram observadas diferenças entre as doses de ONA, consequentemente as baixas dosagens de ONA apresentam a melhor relação custo-benefício.

As formulações de $A B O$ apresentaram maior incidência de efeitos adversos, como redução da força muscular, no entanto, não foram encontradas diferenças estatisticamente significativas entre $A B O$ e ONA. Houve consistência na avaliação do sucesso do tratamento em todos os estudos avaliados (JEE SA, et al., 2015; CAMPANATI A, et al., 2014; DRESSLER D, et al., 2013; IBRAHIM O, et al., 2013; RYSTEDT A, et al., 2013; ALMEIDA ART, et al., 2007; GLOGAU RG, 2007; TALARICO-FILHO S, et al., 2007; MOREAU MS, et al., 2003; ODDERSON IR, 2002; SAADIA D, et al., 2001 NAUMANN M e LOWE NJ, 2001; SCHNIDER P, et al., 1999; SCHNIDER P, et al., 1997).

REAS/EJCH | Vol. 12(12) | e5092 | DOI: https://doi.org/10.25248/reas.e5092.2020 Página 9 de 11 
Poucos efeitos adversos foram relatados nos estudos e a redução da força muscular foi a mais citada. A fraqueza muscular transitória teve uma incidência maior na $A B O$, provavelmente por maior área de superfície de ação do medicamento (FREVERT J, 2015). Em um estudo pacientes relataram resolução completa de queixas sobre fraqueza muscular após uma semana. Embora esses tenham apresentado redução de escore muscular avaliada por dinamômetro nesse mesmo período, o que indica a existência de um nível de fraqueza muscular imperceptível ao paciente.

Além disso, a reaplicação da toxina não é contraindicada devido a sua pequena dosagem e este efeito não parece ser exacerbado em novas aplicações. O que difere de suas contrapartes cosméticas para o tratamento de rugas e expressões faciais que utilizam altas concentração de toxinas botulínicas (HARII K, et al., 2017; BAUMANN L, et al., 2016). Os relatos de redução da força muscular observados nos estudos não foram considerados suficientes para impactar negativamente as atividades diárias, mesmo nas injeções de toxina para hiperidrose palmar, onde a força de pinça do índex-hálux apresentou-se reduzida.

Dor transitória após 24 horas de tratamento foi relatada em alguns estudos e controlada com analgesia simples. A neuralgia transitória teve maior incidência nas aplicações $A B O$, sem diferenças estatísticas entre $A B O$ e ONA. O período de acompanhamento foi considerado insuficiente na maioria dos estudos. Apenas sete estudos realizaram um acompanhamento mínimo suficiente para rastrear o pico do efeito do tratamento (JEE SA, et al., 2015; DRESSLER D, et al., 2013; IBRAHIM O, et al., 2013; ALMEIDA ART, et al., 2007; MOREAU MS, et al., 2003; ODDERSON IR, 2002; SAADIA D, et al., 2001).

Ibrahim O, et al. (2013) exibiram efeitos terapêuticos consistentes de toxina por pelo menos 6 meses. A avaliação de acompanhamento não pôde ser eficaz devido aos dados inconsistentes entre os estudos, e pesquisas futuras devem visar uma avaliação por períodos mais longos, de preferência acima de 6 meses. As aplicações ABO ou ONA não produziram inflamação e/ou infecção. Enquanto a sudorese compensatória (SC) foi relatada em quatro estudos, porém nenhum deles classificou sua intensidade usando a escala HDSS, Minor Tests ou gravimetria. Apenas Odderson IR, et al. (2002) relataram a intensidade. Os principais locais afetados pela SC foram a região lombar, lado interno dos membros inferiores e abdome.

Técnicas cirúrgicas utilizadas para tratar a hiperidrose, como simpatectomias, ablações percutâneas e embolização ganglionar, têm alta incidência de SC, podendo causar desconforto ao paciente. A toxina botulínica, portanto, é uma opção adicional, porém com ação temporaria, no tratamento da hiperidrose primária com pouco efeitos colaterais e baixo índice de sudorese compensatória, podendo ser reaplicada sem aumentar os efeitos adversos.

\section{CONSIDERAÇÕES FINAIS}

Injeções de toxina botulínica são seguras e eficazes para o tratamento da Hiperidrose Primária (Nível de Evidência 1b). Injeções de toxinas atuam como uma medida paliativa, promovendo a cessação ou redução do suor com duração de efeito, extremamente variável, de 4 semanas a 6 meses. A fraqueza muscular transitória é o efeito adverso mais frequente, porém não interfere nas atividades diárias, na primeira aplicação, e as reaplicações de toxina não são contraindicadas. Variação da dose não produz diferenças significativas, e as baixas doses de ONA parecem ter maior custo-benefício. Anestesia local ou bloqueio anestésico são medidas eficazes para controlar o desconforto e a dor, durante o procedimento. Diferente das técnicas permanentes, como as simpatectomias, com altas taxas de sudorese compensatória, as injeções de ONA e ABO produzem baixos níveis desse efeito adverso, exibindo uma incidência de 1,7\% em 576 pacientes.

\section{REFERÊNCIAS}

1. ADAR R, et al. Palmar hyperhidrosis and its surgical treatment: a report of 100 cases. Ann Surg, 1977; 186(1):34.

2. ALMEIDA ART, et al. Pilot Study Comparing the Diffusion of Two Formulations of Botulinum Toxin Type A in Patients with Forehead Hyperhidrosis. Dermatol Surg. 2007;33(s1):S37-43.

3. ASIK ZS, et al. Sympathetic radiofrequency neurolysis for unilateral lumbar hyperhidrosis: a case report. Agri Agri Algoloji Derneginin Yayin Organidir J Turk Soc Algol. 2008;20(3):37-9.

4. BARONCELLO JB, et al. Evaluation of quality of life before and after videothoracoscopic simpathectomy for primary hyperhidrosis. Rev Colégio Bras Cir. 2014;41(5):325-30.

5. BAUMANN L, et al. Duration of Clinical Efficacy of OnabotulinumtoxinA in Crow's Feet Lines: Results from Two Multicenter, Randomized, Controlled Trials. Dermatol Surg. 2016;42(5):598-607. 
6. CAMPANATI A, et al. Onabotulinumtoxin type $A$ (Botox $\AA$ ) versus Incobotulinumtoxin type $A($ Xeomin $\AA$ ) in the treatment of focal idiopathic palmar hyperhidrosis: results of a comparative double-blind clinical trial. J Neural Transm. 2014;121(1):21-6.

7. DIAS LIDEN, et al. Relationship between anxiety, depression and quality of life with the intensity of reflex sweating after thoracoscopic sympathectomy for treatment of primary hyperhidrosis. Rev Colégio Bras Cir. 2016;43(5):354-9.

8. DRESSLER D, ADIB SABERI F. Towards a dose optimisation of botulinum toxin therapy for axillary hyperhidrosis: comparison of different Botox® doses. J Neural Transm. 2013;120(11):1565-7.

9. FELINI R, et al. Prevalência de hiperidrose em uma amostra populacional de Blumenau-SC, Brasil. Anais Brasileiros de Dermatologia, 2009; 84(4): 361-366.

10. FREVERT J. Pharmaceutical, Biological, and Clinical Properties of Botulinum Neurotoxin Type A Products. Drugs RD. $2015 ; 15(1): 1-9$.

11. GLOGAU RG. Topically Applied Botulinum Toxin Type A for the Treatment of Primary Axillary Hyperhidrosis: Results of a Randomized, Blinded, Vehicle-Controlled Study. Dermatol Surg. 2007;33(s1):S76-80.

12. GOLAN DE, et al. Princípios de Farmacologia: a base fisiopatológica da farmacoterapia. $2^{\underline{a}}$ edição. Guanabara, Rio de Janeiro, 2009.

13. HARII K, et al. OnabotulinumtoxinA (Botox) in the Treatment of Crow's Feet Lines in Japanese Subjects. Aesthetic Plast Surg. 2017;41(5):1186-97.

14. HOORENS I, ONGENAE K. Primary focal hyperhidrosis: current treatment options and a step-by-step approach. J Eur Acad Dermatol Venereol. 2012;26(1):1-8.

15. IBRAHIM O, et al. The comparative effectiveness of suction-curettage and onabotulinumtoxin-A injections for the treatment of primary focal axillary hyperhidrosis: A randomized control trial. J Am Acad Dermatol. 2013;69(1):88-95.

16. JEE SA, et al. Comparison of OnabotulinumtoxinA and RimabotulinumtoxinB for the Treatment of Axillary Hyperhidrosis: Dermatol Surg. 2015;41(8):960-7.

17. LAKRAJ AAD, et al. Hyperhidrosis: anatomy, pathophysiology and treatment with emphasis on the role of botulinum toxins. Toxins. 2013;5(4):821-840.

18. LIMA SO, et al. Prevalência de hiperidrose primária e fatores associados em uma capital do nordeste do Brasil: estudo baseado em população. Revista Eletrônica Acervo Saúde, 2020; 12(9): e3815-e3815.

19. LIMA SO, et al. Perfil Epidemiológico e Qualidade de Vida dos Estudantes de Medicina Portadores de Hiperidrose Primária. Revista Brasileira de Educação Médica, 2019; 43(1), 386-394.

20. LOUREIRO M, et al. Hyperhidrosis. Chan: Springer; 2018.

21. MINOR V. Ein neues Verfahren zu der klinischen Untersuchung der Schweissabsonderung. Dtsch Z Für Nervenheilkd. 1928;101(1):302-8.

22. MOREAU MS, et al. A double-blind, randomized, comparative study of DysportÒ vs. BotoxÒ in primary palmar hyperhidrosis. Br J Dermatol. 2003;5.

23. NAUMANN M e LOWE NJ. Botulinum toxin type A in treatment of bilateral primary axillary hyperhidrosis: randomised, parallel group, double blind, placebo controlled trial. Bmj. 2001; 323(7313):596.

24. ODDERSON IR. Long-Term Quantitative Benefits of Botulinum Toxin Type A in the Treatment of Axillary Hyperhidrosis. Dermatol Surg. 2002;4.

25. PATI D e LORUSSO LN. How to Write a Systematic Review of the Literature. HERD. 2018;11(1):15-30.

26. RIEGER R. Management of Plantar Hyperhidrosis with Endoscopic Lumbar Sympathectomy. Thorac Surg Clin. 2016;26(4):465-9.

27. ROMERO RF, et al. Palmar hyperhidrosis: clinical, pathophysiological, diagnostic and therapeutic aspects. Anais Brasileiros de Dermatologia, 2016; 91(6): 716-725.

28. RYSTEDT A, et al. Effect of Botulinum Toxin Concentration on Reduction in Sweating: A Randomized, Double-blind Study. Acta Derm Venereol. 2013;93(6):674-8.

29. SAADIA D, et al. Botulinum toxin type $A$ in primary palmar hyperhidrosis: Randomized, single-blind, two-dose study. Neurology. 2001;57(11):2095-9.

30. SCHLESSINGER J, et al. New Uses of AbobotulinumtoxinA in Aesthetics. Aesthet Surg J. 2017;37(suppl 1):s45-58.

31. SCHNEIER FR, et al. Social anxiety and functional impairment in patients seeking surgical evaluation for hyperhidrosis. Compr Psychiatry, 2012;53(8):1181-6.

32. SCHNIDER $P$, et al. A randomized, double-blind, placebo-controlled trial of botulinum $A$ toxin for severe axillary hyperhidrosis. Br J Dermatol. 1999;140(4):677-80.

33. SCHNIDER $P$, et al. Double-blind trial of botulinum A toxin for the treatment of focal hyperhidrosis of the palms. Brit $J$ Dermatol. 1997;136(4):548-552.

34. SOLISH N, et al. A Comprehensive Approach to the Recognition, Diagnosis, and Severity-Based Treatment of Focal Hyperhidrosis: Recommendations of the Canadian Hyperhidrosis Advisory Committee. Dermatol Surg. 2007;33(8):908-23.

35. TALARICO-FILHO S, et al. A Double-Blind, Randomized, Comparative Study of Two Type A Botulinum Toxins in the Treatment of Primary Axillary Hyperhidrosis. Dermatol Surg. 2007;33(s1):S44-50.

36. VASCONCELLOS RC, et al. Updates on the cosmiatric and therapeutic use of botulinum toxin. Surg Cosmet Dermatol. 2019; 11(2):97-104.

37. VIECHTBAUER W. Conducting Meta-Analyses in $R$ with the metafor Package. J Stat Softw. 2010;36(3).

38. WEBER A, et al. Psychosocial aspects of patients with focal hyperhidrosis. Marked reduction of social phobia, anxiety and depression and increased quality of life after treatment with botulinum toxin A. Br J Dermatol. 2005;152(2):3425. 